\title{
5G Enhanced Mobile Broadband (eMBB): Evaluation of Scheduling Algorithms Performances for Time-Division Duplex Mode
}

\author{
https://doi.org/10.3991/ijim.v16i01.25941 \\ Asmae Mamane ${ }^{1(\otimes)}$, M. Fattah ${ }^{2}$, M. El Ghazi ${ }^{1}$, M. El Bekkali ${ }^{1}$ \\ ${ }^{1}$ Data Sciences and Emerging Systems, Sidi Mohammed Ben \\ Abdellah University, Fez, Morocco \\ ${ }^{2}$ IMAGE Laboratory, Moulay Ismail University, Meknes, Morocco \\ asmae.mamane@gmail.com
}

\begin{abstract}
G mobile communications introduce novel solutions to overcome the frequency spectrum's shortage. It broadens the spectrum band to millimeter-waves, employs multiple numerologies to calculate subcarrier spacing, and supports various division duplex modes. Furthermore, the fifth generation of mobile networks intends to employ both frequency division duplex and time division duplex. This study focuses on Time Division Duplex (TDD) mode. Compared to the Frequency Division Duplex (FDD), the time duplex mode enhances flexibility and allows efficient frequency spectrum usage. However, the recent papers addressing resource scheduling issues for TDD duplex employ only the current classical schedulers, which were primarily designed for FDD mode, to accomplish radio resource allocation. In this paper, we compared the achievable throughput and data accumulated in the buffer of these schedulers to assess their suitability and compatibility with TDD specifications. The resulting performances show that an appropriate scheduler in line with TDD requirements should be implemented to exploit the available spectrum efficiently and reach the required throughput.
\end{abstract}

Keywords - time division duplex, scheduling, throughput, radio resource allocation, 5G NR

\section{Introduction}

Across the successive generations of mobile networks, radio resource management and optimal frequency spectrum utilization have always been the most crucial topics to tackle [1]. Considering that high spectrum availability is essential to accommodate the rising demand of bandwidth to fulfill the end-user needs, the fifth generation of mobile networks $(5 \mathrm{G})$ employs unlicensed, licensed, and shared spectrum. In addition, it expands the spectrum to low bands below $1 \mathrm{GHz}$ and goes beyond $24 \mathrm{GHz}$ by introducing the mmWaves [2]-[4].

Furthermore, 5G NR introduces numerologies $\mu=\{0,1,2,3\}$ for allowing flexible and efficient spectrum usage. The numerologies define the subcarrier spacing to 
employ $\mathrm{SCS}=2^{\wedge} \mu^{*} 15 \mathrm{kHz}$, the number of slots of a subframe, and the number of resource blocks available for each bandwidth [5].

Moreover, 5G supports various use cases and scenarios that necessitate customized performance characteristics [6]-[8]. Many aspects must be considered to meet the quality-of-service (QoS) criteria established for $5 \mathrm{G}$, such as the duplex mode, the subcarrier spacing (SCS), and the scheduling schemes. Therefore, using the proper division duplex mode is critical for any communication system [9], [10].

According to the 3GPPP reports detailed in [11]-[13], the frequency spectrum adopted for 5G NR is divided into two frequency ranges, FR1 and FR2, named sub 6GHz and mm waves, respectively. The FR1 supports both duplex modes, FDD and TDD, by implementing three numerologies, namely $15 \mathrm{khz}, 30 \mathrm{khz}$, and $60 \mathrm{khz}$. However, the FR2 operates only on TDD mode using $60 \mathrm{khz}$ and $120 \mathrm{khz}$ subcarrier spacing (SCS).

A literature analysis indicates that almost all studies addressing radio resource management and spectrum usage adopt frequency division duplex [14]-[18]. Besides, the works [19], [20] published recently and addressing the time division duplex employ the classical scheduler, the Proportional Fair (PF), to ensure resource allocation for the users. Based on these works, we decided to study the suitability of the classical schedulers, namely Round Robin (RR), Best CQI(BCQI), and Proportional Fair (PF), for the TDD mode.

In this research, we conducted a thorough investigation to compare and assess the performance of the scheduling algorithms mentioned previously on TDD. We opted for various types of traffic and different SCS values for the enhanced mobile broadband (eMBB) users to provide a clear overview of the algorithms under consideration. We analyzed the performances of three types of traffics, heavy download as the video streaming service, heavy uplink by storing data on the cloud, and a balanced traffic use case through a video conference scenario.

The remainder of this work is structured as follows: Section 2 describes and compares the duplex modes, Section 3 outlines the scenarios used to carry out this study, Section 4 gives the simulation findings and discusses the performance in terms of achievable throughput and the accumulated data in the buffer for each case, and Section 5 summarizes the article.

\section{Duplex modes}

The fifth generation of mobile networks supports both duplexing FDD and TDD since it introduces various use cases and services demanding different requirements. Moreover, it deploys the same frame structure for paired and unpaired modes, compared to LTE networks which employ different frame structures, type 1 and type 2, to perform FDD and TDD duplexing, respectively [21].

\subsection{FDD and TDD modes specifications}

The Frequency Division Duplex (FDD) mode grants a simultaneous data transfer over a paired spectrum by assigning different frequency bands to upstream and downstream traffics. It separates the two frequencies by a guard band to avoid the 
interferences between transmission and reception signals. The sent and received packets are transmitted through and by the same antenna. Thus, using a duplexer remains essential to distinguish the traffic frequency. The frequency division duplex provides balanced bandwidths to ensure packets transfer from and to the end-user. Therefore, the FDD mode allows symmetric and simultaneous communications [22].

Though, the Time Division Duplex (TDD) transmits uplink and downlink packets via the unpaired spectrum. Furthermore, the TDD duplex mode sends each stream in separate time slots to prevent interferences since the uplink and downlink share the same frequency carrier. Hence, the sent and received packets are asynchronous. A split time is necessary for the unpaired mode to allow the communicating device to switch from the sending mode to receiving and vice versa. The switching time relies upon the distance separating the UE from the gNB. Besides, the time gap that separates the up and down streams should be insignificant to provide approximately synchronous bidirectional flow. Thus, the mmWaves band employed in small cells operates on TDD mode to prevent the QoS decrease due to extensive time guard. Moreover, the timedivision duplex is suitable for asymmetric traffics since it dynamically allocates slots to each stream [23]. Table 1 summarizes and compares the duplexing modes supported by $5 \mathrm{G}$ NR.

Table 1. Comparative analysis between the FDD and TDD modes

\begin{tabular}{|l|l|l|}
\hline \multicolumn{1}{|c|}{ Parameter } & \multicolumn{1}{c|}{ FDD } & UDD \\
\hline Spectrum usage & Paired & No \\
\hline A continuous flow of data & Yes & High \\
\hline Spectral efficiency & Low due to large guard band & Asymmetric services \\
\hline Type of service & Symmetric services & $\begin{array}{l}\text { Timing synchronization is highly } \\
\text { required }\end{array}$ \\
\hline Synchronization & No need & Low \\
\hline Coverage & High & Small cells \\
\hline Type of cell & Macrocell and microcell & Medium du synchronization issue \\
\hline Complexity & High due to frequency filter & Low \\
\hline Cost & High due to duplexer use & FR1 and FR2 \\
\hline Frequency range & FR1 & Handled \\
\hline MIMO and Beamforming & Not applicable & Yes \\
\hline Dynamic and flexible & No & \\
\hline
\end{tabular}

\subsection{The difference between LTE TDD and NR TDD}

A TDD frame is $10 \mathrm{~ms}$ long and consists of either DL, UL, or Special (S) slot with a $1 \mathrm{~ms}$ duration. In LTE, The $\mathrm{S}$ sub-frame performs switching from downstream to upstream. For $5 \mathrm{G}$ NR, the symbols of special slot (S) can be configured as a DL symbol, a UL symbol, or a flexible symbol denoted (F).

Besides, the LTE TDD supports only seven static patterns for DL-UL frame configuration [24]. On the contrary, 5G NR TDD keeps changing the frame configuration 
to meet the fluctuating uplink/downlink traffic needs. In addition, the 5G NR defines different DL-UL periodicities that vary depending on the SCS adopted. In other words, it enables dynamic frame size for each numerology.

Moreover, the 5G TDD enables more than 56 slot format patterns to configure the special slot $(\mathrm{S})$ symbols to allow a very flexible configuration and improve overall throughput. The flexible symbols denoted F are instantaneously adjusted to UL or DL symbols depending on the volume and type of traffic.

In addition, the time duplexing in 5G NR supports symbol-based scheduling instead of slot-based scheduling used for LTE TDD and FDD. Symbol-based scheduling performs and updates the scheduling processes in every slot, which affords a short transmission duration spanning a few symbols in the slot. Hence 5G NR TDD using symbol-based scheduling improves spectral efficiency and frame flexibility [25], [26].

Figure 1 demonstrates a 5G NR TDD radio frame. For numerology $\mu=2$ (SCS $=$ $60 \mathrm{khz}$ ) the subframe is divided into 40 slots of $0.25 \mathrm{~ms}$ as detailed in [25]. Every 5 slots represent a DL-UL periodicity of $1.25 \mathrm{~ms}$. In this example, symbols of the special slot (S) are configured conforming to the $\mathrm{n} 27$ slot format.

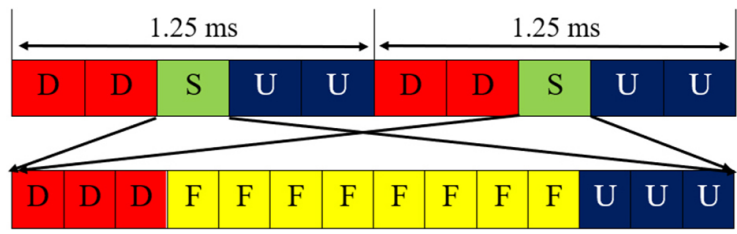

Fig. 1. Special slot configuration

\section{Research method}

This paper aims to compare schedulers' performances for enhanced mobile broadband communications. Our simulations opted for an ultra-dense urban environment where 10 users transmit their traffics simultaneously and continuously through a microcell [27]. In line with the 3 GPP requirements, we employ a 400-meter-radius small cell [28], [29].

Furthermore, various parameters depending on traffic type, frequency range, and SCS must be defined to perform dynamic and flexible TDD scheduling.

Since the TDD mode is suitable for both 5G NR frequency ranges, we evaluated the performances of the schedulers using two frequency bands, namely 41 for the FR1 and n257 for the FR2 [30].

As explained above, the DL-UL periodicity, the number of slots per frame, and the number of resources blocks depend on the SCS adopted. In this study, we employed three different durations for the DL-UL periodicity, $5 \mathrm{~ms}, 1.25 \mathrm{~ms}$, and $0.625 \mathrm{~ms}$ considering $30 \mathrm{kHz}, 60 \mathrm{kHz}$, and $120 \mathrm{kHz}$ SCS, respectively. As stated in section 38.213-11.1 of [25], each combination of DL-UL periodicity and a subcarrier value provides the number of slots in the subframe. To achieve the best performances through the compared algorithms, we choose, based on the 3GPP specifications, the highest number of 
resource blocks for the numerologies studied $273 \mathrm{RB}$ for $30 \mathrm{kHz}$ SCS and 264RB for both $60 \mathrm{kHz}$ and $120 \mathrm{kHz}$ [11], [12].

Besides, we configured the special slot (S), conforming to Table 11.1.1-1 in [25]. We opted for three-slot formats n27, n28, and n34 to address balanced load traffic (VoIP), Heavy DL traffic (Video streaming), and Heavy UL traffic (cloud storage, FTP), respectively. Therefore, we set up the parameters of the different scenarios as recommended in section 11 of the technical report cited in [25] and summarized in Table 2.

Table 2. Simulation scenario [25]

\begin{tabular}{|l|c|c|c|c|c|c|c|c|c|}
\hline \multicolumn{1}{|c|}{$\begin{array}{c}\text { Traffic } \\
\text { Type }\end{array}$} & \multicolumn{3}{|c|}{ Heavy DL } & \multicolumn{3}{c|}{ Balanced Load } & \multicolumn{3}{c|}{ Heavy UL } \\
\hline $\begin{array}{l}\text { Frequency } \\
\text { range }\end{array}$ & $\begin{array}{c}\text { FR1 } \\
(30 \mathrm{khz})\end{array}$ & $\begin{array}{c}\text { FR2 } \\
(60 \mathrm{khz})\end{array}$ & $\begin{array}{c}\text { FR2 } \\
(120 \mathrm{khz})\end{array}$ & $\begin{array}{c}\text { FR1 } \\
(30 \mathrm{khz})\end{array}$ & $\begin{array}{c}\text { FR2 } \\
(60 \mathrm{khz})\end{array}$ & $\begin{array}{c}\text { FR2 } \\
(120 \mathrm{khz})\end{array}$ & $\begin{array}{c}\text { FR1 } \\
(30 \mathrm{khz})\end{array}$ & $\begin{array}{c}\text { FR2 } \\
(60 \mathrm{khz})\end{array}$ & $\begin{array}{c}\text { FR2 } \\
(120 \mathrm{khz})\end{array}$ \\
\hline $\begin{array}{l}\text { Frequency } \\
\text { band }\end{array}$ & $\mathrm{n} 41$ & $\mathrm{n} 257$ & $\mathrm{n} 257$ & $\mathrm{n} 41$ & $\mathrm{n} 257$ & $\mathrm{n} 257$ & $\mathrm{n} 41$ & $\mathrm{n} 257$ & $\mathrm{n} 257$ \\
\hline Bandwidth & $100 \mathrm{MHz}$ & $200 \mathrm{MHz}$ & $400 \mathrm{MHz}$ & $100 \mathrm{MHz}$ & $200 \mathrm{MHz}$ & $400 \mathrm{MHz}$ & $100 \mathrm{MHz}$ & $200 \mathrm{MHz}$ & $400 \mathrm{MHz}$ \\
\hline SCS & $30 \mathrm{KHz}$ & $60 \mathrm{Khz}$ & $120 \mathrm{KHz}$ & $30 \mathrm{KHz}$ & $60 \mathrm{Khz}$ & $120 \mathrm{KHz}$ & $30 \mathrm{KHz}$ & $60 \mathrm{Khz}$ & $120 \mathrm{KHz}$ \\
\hline RB & 273 & 264 & 264 & 273 & 264 & 264 & 273 & 264 & 264 \\
\hline $\begin{array}{l}\text { Periodicity } \\
\text { (ms) }\end{array}$ & 5 & 1.25 & 0.625 & 5 & 1.25 & 0.625 & 5 & 1.25 & 0.625 \\
\hline $\begin{array}{l}\text { Number of } \\
\text { slots }\end{array}$ & 10 & 5 & 5 & 10 & 5 & 5 & 10 & 5 & 5 \\
\hline $\begin{array}{l}\text { Number } \\
\text { lots DL }\end{array}$ & 6 & 2 & 2 & 4 & 2 & 2 & 2 & 1 & 1 \\
\hline $\begin{array}{l}\text { Number } \\
\text { slots UL }\end{array}$ & 1 & 1 & 1 & 4 & 2 & 2 & 6 & 2 & 2 \\
\hline $\begin{array}{l}\text { Number } \\
\text { symbols DL }\end{array}$ & 12 & 12 & 12 & 3 & 3 & 3 & 1 & 1 & 1 \\
\hline $\begin{array}{l}\text { Number } \\
\text { symbols UL }\end{array}$ & 1 & 1 & 1 & 3 & 3 & 3 & 12 & 12 & 12 \\
\hline
\end{tabular}

\section{Simulation results and discussion}

In this section, we analyze the simulation results of traditional scheduling techniques (Round Robin [31], best CQI [32], and proportional fair [33]). The simulation results are evaluated through their throughput and the size of the remaining data in the buffer.

\subsection{Balanced load traffic: VoIP}

As depicted in Figures 2 and 3, the peak data attains $316.75 \mathrm{Mbps}$, 620Mbps, and $1.24 \mathrm{Gbps}$ for $100 \mathrm{MHz}, 200 \mathrm{MHz}$, and $400 \mathrm{MHz}$, respectively. Furthermore, the Best CQI achieves the greatest downstream traffic throughput value of $288.98 \mathrm{Mbps}$ in FR2. Hence, the uplink and downlink throughput performed by the evaluated schedulers present only $23 \%$ of the peak data rate that may be reached using the same simulation parameters. 


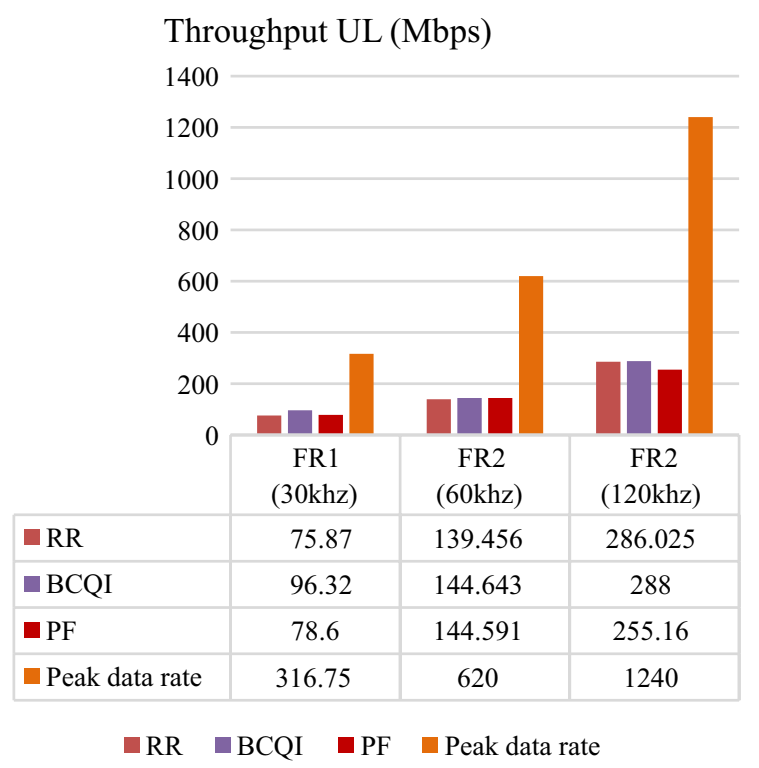

Fig. 2. Uplink throughput for VoIP traffic

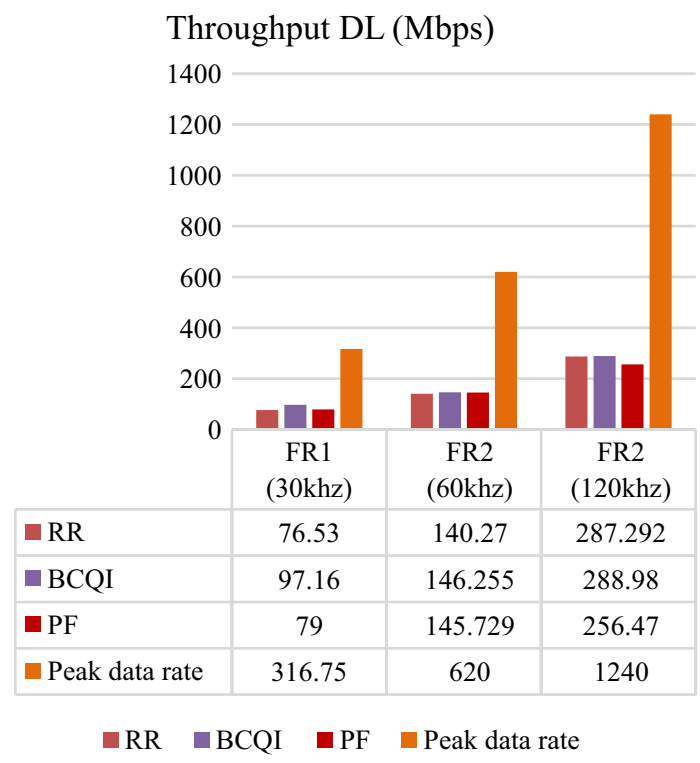

Fig. 3. Downlink throughput for VoIP traffic 
However, at the end of the simulation, the buffer size exceeds $9154 \mathrm{KBs}$, as shown in Table 3.

Table 3. Remaining data in the buffer for UL and DL balanced traffic

\begin{tabular}{|c|c|c|c|c|c|c|}
\hline & \multicolumn{3}{|c|}{ Buffer DL (KBs) } & \multicolumn{3}{c|}{ Buffer UL (KBs) } \\
\hline Scheduler & FR1 30khz & FR2 60khz & FR2 120khz & FR1 30khz & FR2 60khz & FR2 120khz \\
\hline RR & 9138.8 & 9132.54 & 9130.21 & 9143.51 & 9141.73 & 9136.39 \\
\hline BCQI & 9154.3 & 9136.56 & 9124.34 & 9156.26 & 9126.05 & 9122.34 \\
\hline PF & 9141.84 & 9135.68 & 9130.5 & 9143.46 & 9138.7 & 9134.02 \\
\hline
\end{tabular}

\subsection{Heavy downlink traffic: video streaming}

In this scenario, we focus only on the Downlink performances. Figure 4 displays the data rates achieved by the three classical schedulers mainly developed to serve FDD traffics. We notice that the best CQI attains the highest value for the three bandwidth sizes. The best CQI delivers a $151.8 \mathrm{Mbps}$ data rate compared to $465 \mathrm{Mbps}$ peak data rate using $100 \mathrm{MHz}$ bandwidth, for $200 \mathrm{MHz}$ of bandwidth, it exceeds $218 \mathrm{Mbps}$ compared to $750 \mathrm{Mbps}$ peak level, and we note $518.58 \mathrm{Mbps}$ achieved by the best CQI whereas the max data rate surpasses $1.5 \mathrm{Gbps}$ for $400 \mathrm{MHz}$ bandwidth.

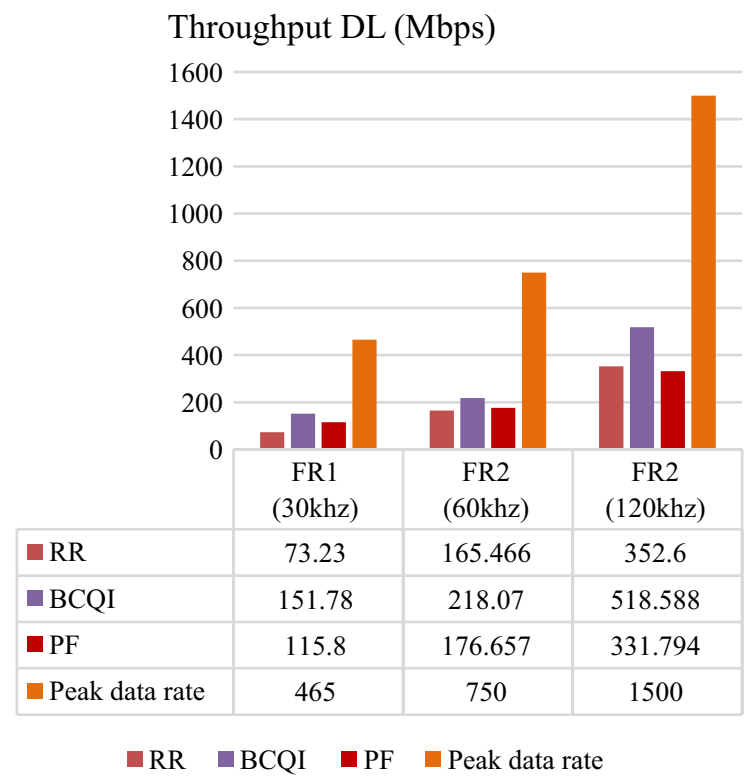

Fig. 4. Downlink throughput for video streaming traffic

As a result, the ratio between the highest throughput value, provided by the best CQI, and the peak data rate achieved does not reach $35 \%$ for video streaming. 
Meanwhile, Table 4 shows that the accumulated buffer data is around $9135 \mathrm{KBs}$ for all the schedulers performing in three numerologies and bandwidths.

Table 4. Remaining data in the buffer for heavy DL traffic

\begin{tabular}{|l|c|c|c|}
\hline & \multicolumn{3}{|c|}{ Buffer DL (KBs) } \\
\hline \multicolumn{1}{|c|}{ Scheduler } & FR1 (30khz) & FR2 (60khz) & FR2 (120khz) \\
\hline RR & 9146.13 & 9139.56 & 9136.4 \\
\hline BCQI & 9138.1 & 9125.69 & 9120.59 \\
\hline PF & 9149.57 & 9136.84 & 9128.53 \\
\hline
\end{tabular}

\subsection{Heavy uplink traffic: cloud storage}

By analyzing the uplink performances in Figure 5 and Table 5, we recognize that buffer data outpaces $9130 \mathrm{KBs}$, yet the throughput is restricted to $600 \mathrm{Mbps}$ for the best CQI. In comparison, the Round Robin's throughput fluctuates between $85 \mathrm{Mbps}$ and $340 \mathrm{Mbps}$. Aside from that, the PF rate level is bound to $450 \mathrm{Mbps}$.

The peak data rate, on the other hand, exceeds $1500 \mathrm{Mbps}$. Thus, none of the preceding algorithms achieves $39 \%$ of the expected throughput for this use case.

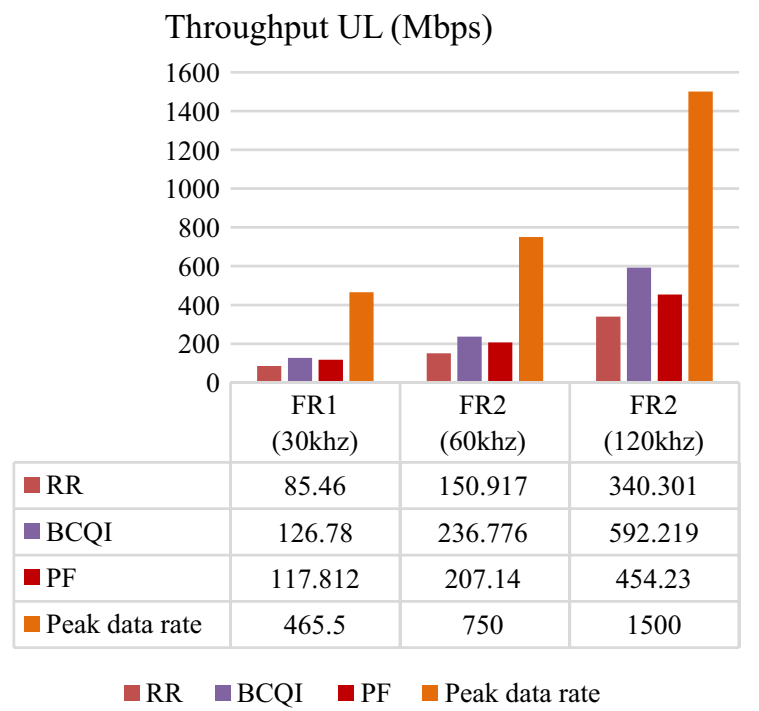

Fig. 5. Uplink throughput for cloud storage traffic 
Table 5. Remaining data in the buffer for heavy UL traffic

\begin{tabular}{|l|c|c|c|}
\hline & \multicolumn{3}{|c|}{ Buffer UL (KBs) } \\
\hline \multicolumn{1}{|c|}{ Scheduler } & FR1 (30khz) & FR2 (60khz) & FR2 (120khz) \\
\hline RR & 9141.77 & 9139.52 & 9121.34 \\
\hline BCQI & 9130.24 & 9129.36 & 9109.36 \\
\hline PF & 9132.75 & 9131.35 & 9119.09 \\
\hline
\end{tabular}

As shown in Figures 2 and 3, the peak data rates for the three SCSs adopted are the same when comparing uplink and downlink, owing to the balanced load traffic and the fact that we configured the same number of slots and symbols for uplink and downlink. However, video streaming and cloud upload services experience greater peak data rates than VoIP since they transmit in just one way.

In all cases, we found out that in the best situations, the ratio between the peak data rate and the achieved throughput that we opted for evaluating the scheduling algorithms can barely reach $39 \%$. Besides, the simulation results reveal that the accumulated data in the buffer range between $9120 \mathrm{KBs}$ and $9150 \mathrm{KBs}$ for the three scenarios, indicating that at the end of the simulations, there is still some data to transmit even though all of the RBs have been utilized. This implies that the RBs are not efficiently allocated to the users through the evaluated schedulers.

\section{Conclusion}

This work studied the duplex modes supported by $5 \mathrm{G} \mathrm{NR}$, namely frequency division duplex and time division duplex. Furthermore, this work underlines the main differences between LTE and 5G NR TDD modes.

In addition, we performed a comparative performance evaluation of the classical schedulers developed mainly for FDD to assess their applicability and compatibility with dynamic TDD criteria. Hence, we selected distinct types of traffic and SCS values for enhanced mobile broadband (eMBB) users to provide a comprehensive understanding of the algorithms under consideration. Three types of traffic have been examined namely video streaming, data upload in the cloud, and a video conference scenario to evaluate balanced load traffic.

The results demonstrate that the scheduling algorithms used to perform resource allotting for time division duplex present only $39 \%$ of the peak data rate achieved in the same circumstances. Therefore, we conclude that these algorithms are unsuitable for flexible TDD scheduling.

As future work, we are working on a new scheduler considering the specifications of flexible TDD to meet the required throughput and reach the QoS requirements set by $5 \mathrm{G}$. 


\section{Acknowledgment}

This work is supported by the CNRST (National Center for Scientific and Technical Research) via the Research Excellence Scholarships Program.

\section{$7 \quad$ References}

[1] A. el Mahjoubi, T. Mazri, and N. Hmina, "NB-IoT and eMTC: Engineering results towards 5G/IoT mobile technologies," International Journal of Interactive Mobile Technologies, vol. 13, no. 1, pp. 115-126, 2019, doi: https://doi.org/10.3991/ijim.v13i01.9728

[2] GSMA, "5g Spectrum - GSMA public policy position,” Public Policy Position, 2021.

[3] A. Es-Saqy et al., "28 GHz balanced pHEMT VCO with low phase noise and high output power performance for $5 \mathrm{G}$ mm-wave systems," International Journal of Electrical and Computer Engineering, vol. 10, no. 5, pp. 4623-4630, 2020, doi: https://doi.org/10.11591/ ijece.v10i5.pp4623-4630

[4] B. Younes, F. Mohammed, M. Saïd, and M. el Bekkali, "5G uplink interference simulations, analysis and solutions: The case of pico cells dense deployment," International Journal of Electrical and Computer Engineering (IJECE), vol. 11, no. 3, pp. 2245-2255, Jun. 2021, doi: https://doi.org/10.11591/ijece.v11i3.pp2245-2255

[5] Technical Specification, "TS 138211 - V16.3.0 - 5G; NR; Physical channels and modulation (3GPP TS 38.211 version 16.3.0 Release 16),” 2020. Accessed: Feb. 19, 2021. [Online]. Available: https://portal.etsi.org/TB/ETSIDeliverableStatus.aspx

[6] I. Omirzak, L. N. Gumilyov, Y. Razumova, and S. Nikishina, "Paper-new generation mobile networks and their application in electronic learning new generation mobile networks and their application in electronic learning," International Journal of Interactive Mobile Technologies, vol. 15, no. 2, pp. 157-171, Jan. 2021, doi: https://doi.org/10.3991/ijim.v15i02.18323

[7] A. Mamane, M. el Ghazi, G.-R. Barb, and M. Otesteanu, "5G heterogeneous networks: an overview on radio resource management scheduling schemes," in 2019 7th Mediterranean Congress of Telecommunications (CMT), Oct. 2019, pp. 1-5. doi: https://doi.org/10.1109/ CMT.2019.8931369

[8] A. Mamane, M. Fattah, M. el Ghazi, Y. Balboul, M. el Bekkali, and S. Mazer, "Proportional fair buffer scheduling algorithm for $5 \mathrm{G}$ enhanced mobile broadband," International Journal of Electrical and Computer Engineering, vol. 11, no. 5, pp. 4165-4173, Oct. 2021, doi: https://doi.org/10.11591/ijece.v11i5.pp4165-4173

[9] "Duplex Schemes-NR LTE related tech oriented blog." https://info-nrlte.com/category/ 5g-nr/duplex-schemes/ (accessed Jun. 02, 2021).

[10] A. Mamane, M. el Ghazi, S. Mazer, M. Bekkali, M. Fattah, and M. Mahfoudi, "The impact of scheduling algorithms for real-time traffic in the 5G femto-cells network," in 9th International Symposium on Signal, Image, Video and Communications, ISIVC 2018-Proceedings, Nov. 2018, pp. 147-151. doi: https://doi.org/10.1109/ISIVC.2018.8709175

[11] TSGR, "TS 138 101-2 - V15.5.0 - 5G; NR; User Equipment (UE) radio transmission and reception; Part 2: Range 2 Standalone (3GPP TS 38.101-2 version 15.5.0 Release 15)," 2019. Accessed: May 31, 2021. [Online]. Available: https://portal.etsi.org/TB/ETSIDeliverableStatus.aspx

[12] TSGR, "TS 138 101-1 - V15.5.0 - 5G; NR; User Equipment (UE) radio transmission and reception; Part 1: Range 1 Standalone (3GPP TS 38.101-1 version 15.5.0 Release 15)," 2019. Accessed: May 31, 2021. [Online]. Available: https://portal.etsi.org/TB/ETSIDeliverableStatus.aspx 
[13] 3GPP, "3rd Generation partnership project; technical specification group radio access network; study on lte time division duplex (tdd)-frequency division duplex (fdd) joint operation including carrier aggregation (ca) (release 12).” [Online]. Available: http://www.3gpp.org

[14] S. K. Singh, R. Singh, and B. Kumbhani, "A review on cellular network infrastructure evolution: challenges and opportunities," in 2019 IEEE 14th International Conference on Industrial and Information Systems: Engineering for Innovations for Industry 4.0, ICIIS 2019-Proceedings, Dec. 2019, pp. 163-168. doi: https://doi.org/10.1109/ICIIS47346.2019.9063261

[15] M. Agiwal, A. Roy, N. Saxena, E. Agiwal, A. Roy, and N. Saxena, "Next generation 5g wireless networks: a comprehensive survey," IEEE Communications Surveys Tutorials, vol. 18, no. 3, pp. 1617-1655, 2016, doi: https://doi.org/10.1109/COMST.2016.2532458

[16] T. O. Olwal, K. Djouani, and A. M. Kurien, "A survey of resource management toward $5 \mathrm{~g}$ radio access networks," IEEE Communications Surveys and Tutorials, vol. 18, no. 3, pp. 1656-1686, 2016, doi: https://doi.org/10.1109/COMST.2016.2550765

[17] F. Capozzi, G. Piro, L. A. Grieco, G. Boggia, and P. Camarda, "Downlink packet scheduling in LTE cellular networks: Key design issues and a survey," IEEE Communications Surveys and Tutorials, vol. 15, no. 2, pp. 678-700, 2013, doi: https://doi.org/10.1109/ SURV.2012.060912.00100

[18] F. Capozzi, G. Piro, L. A. Grieco, G. Boggia, and P. Camarda, "Downlink packet scheduling in LTE cellular networks: Key design issues and a survey," IEEE Communications Surveys and Tutorials, vol. 15, no. 2, pp. 678-700, 2013, doi: https://doi.org/10.1109/ SURV.2012.060912.00100

[19] A. A. Esswie and K. I. Pedersen, "On the ultra-reliable and low-latency communications in flexible tdd/fdd 5g networks," Sep. 2020. doi: https://doi.org/10.1109/CCNC46108 .2020 .9045657

[20] J. Lee, M. Rim, and C. G. Kang, "Decentralized slot-ordered cross link interference control scheme for dynamic time division duplexing (tdd) in $5 \mathrm{~g}$ cellular system," IEEE Access, vol. 9, pp. 63567-63579, 2021, doi: https://doi.org/10.1109/ACCESS.2021.3074176

[21] "What duplex-schemes 4G-LTE and 5G-NR networks use: FDD or TDD? - Commsbrief." https://commsbrief.com/what-duplex-schemes-4g-lte-and-5g-nr-networks-use-fdd-or-tdd/ (accessed Jun. 02, 2021).

[22] A. Grami, "Communication networks," in Introduction to Digital Communications, Elsevier, 2016, pp. 457-491. doi: https://doi.org/10.1016/B978-0-12-407682-2.00011-9

[23] “TDD FDD Duplex Schemes » Electronics Notes.” https://www.electronics-notes.com/ articles/connectivity/cellular-mobile-phone/duplex-time-frequency-division-tdd-fdd.php (accessed Jun. 02, 2021).

[24] T. Specification and E. Universal, "TS 136213 - V13.3.0 - LTE; Evolved Universal Terrestrial Radio Access (E-UTRA); Physical layer procedures (3GPP TS 36.213 version 13.3.0 Release 13)," vol. 0, 2016, Accessed: Jun. 14, 2021. [Online]. Available: https://portal.etsi. org/TB/ETSIDeliverableStatus.aspx

[25] 3GPP, “138 213 - V15.8.0 - 5G; NR; Physical layer procedures for control,” 2020. Accessed: Jun. 14, 2021. [Online]. Available: https://portal.etsi.org/TB/ETSIDeliverableStatus.aspx

[26] TSGR, "TS 138331 - V15.5.1 - 5G; NR; Radio Resource Control (RRC); Protocol specification (3GPP TS 38.331 version 15.5.1 Release 15)," vol. 0, 2019, Accessed: Jun. 14, 2021. [Online]. Available: https://portal.etsi.org/TB/ETSIDeliverableStatus.aspx

[27] A. S. Afolabi, S. Ahmed, and O. A. Akinola, "A reinforcement learning approach for interference management in heterogeneous wireless networks," International Journal of Interactive Mobile Technologies, vol. 15, no. 12, pp. 65-85, Jun. 2021, doi: https://doi.org/10.3991/ ijim.v15i12.20751 
[28] G. E. Athanasiadou, P. Fytampanis, D. A. Zarbouti, G. v. Tsoulos, P. K. Gkonis, and D. I. Kaklamani, "Radio network planning towards $5 \mathrm{~g}$ mmwave standalone small-cell architectures," Electronics (Switzerland), vol. 9, no. 2, Feb. 2020, doi: https://doi.org/10.3390/ electronics 9020339

[29] "Small Cell Solutions- Increasing 5G capacity and coverage - ericsson." https://www. ericsson.com/en/small-cells (accessed Jun. 12, 2021).

[30] 3GPP, "5G; Study on New Radio (NR) access technology,” 2018. [Online]. Available: http:// www.etsi.org/standards-search

[31] X. Yuan and Z. Duan, "Fair round-robin: a low complexity packet schduler with proportional and worst-case fairness," IEEE Transactions on Computers, vol. 58, no. 3, pp. 365-379, Mar. 2009, doi: https://doi.org/10.1109/TC.2008.176

[32] M. A. Ibraheem, N. Elshennawy, and A. M. Sarhan, "A proposed modified proportional fairness scheduling (mpf-bcqi) algorithm with best cqi consideration for lte-a networks," Proceedings-2018 13th International Conference on Computer Engineering and Systems, ICCES 2018, vol. 7, pp. 360-368, 2019, doi: https://doi.org/10.1109/ICCES.2018.8639213

[33] T. Dierdre, "Forward Link Multiuser Diversity Through Proportional Fair Scheduling," 1999.

\section{Authors}

Asmae Mamane received her D.I. degree in networks and telecommunications from Hassan I University, Settat, Morocco in 2016. She is now a Ph.D. student in 5G Technology at Artificial Intelligence, Data Sciences, and Emerging Systems Laboratory of Sidi Mohamed Ben Abdellah University Fez Morocco. Her research interests include resource allocation techniques, scheduling algorithms, and improvement of QoS in 5G technology. (email: asmae.mamane@gmail.com).

Mohammed Fattah received his Ph.D. in Telecommunications and CEM at the Sidi Mohamed Ben Abdellah University (USMBA), Fez, Morocco, in 2011. He is a professor in the Electrical Engineering Department of the High school of technology at the Moulay Ismail University (UMI), Meknes, Morocco and he is responsible for the research team 'Intelligent Systems, Networks and Telecommunications', IMAGE laboratory, UMI. (email: m.mohammedfattah@umi.ac.ma).

Mohammed El Ghazi is a professor in the Electrical and Computer Engineering Department of Superior School of Technology at the Sidi Mohamed Ben Abdellah University (USMBA), Fez, Morocco. He is a member of the Laboratory of Artificial Intelligence, Data Sciences, and Emerging Systems. (email: mohammed.elghazi@usmba. ac.ma).

Moulhime EI Bekkali Moulhime El Bekkali is a professor in the National School of Applied Sciences at the Sidi Mohamed Ben Abdellah University (USMBA), Fez, Morocco. He is a member of the Laboratory of Artificial Intelligence, Data Sciences, and Emerging systems. (email: moulhime.elbekkali@usmba.ac.ma).

Article submitted 2021-08-02. Resubmitted 2021-10-06. Final acceptance 2021-10-09. Final version published as submitted by the authors. 